\title{
Clinical and genetical heterogeneity of late-onset multiple acyl-coenzyme A dehydrogenase deficiency
}

\author{
Sarah C Grünert
}

\begin{abstract}
Background: Multiple acyl-CoA dehydrogenase deficiency (MADD) is an autosomal recessive disorder caused by deficiency of electron transfer flavoprotein or electron transfer flavoprotein dehydrogenase. The clinical picture of late-onset forms is highly variable with symptoms ranging from acute metabolic decompensations to chronic, mainly muscular problems or even asymptomatic cases.

Methods: All 350 cases of late-onset MADD reported in the literature to date have been analyzed and evaluated with respect to age at presentation, diagnostic delay, biochemical features and diagnostic parameters as well as response to treatment.

Results: Mean age at onset was 19.2 years. The mean delay between onset of symptoms and diagnosis was 3.9 years. Chronic muscular symptoms were more than twice as common as acute metabolic decompensations ( $85 \%$ versus $33 \%$ of patients, respectively). $20 \%$ had both acute and chronic symptoms. $5 \%$ of patients had died at a mean age of 5.8 years, while $3 \%$ of patients have remained asymptomatic until a maximum age of 14 years. Diagnosis may be difficult as a relevant number of patients do not display typical biochemical patterns of urine organic acids and blood acylcarnitines during times of wellbeing. The vast majority of patients carry mutations in the ETFDH gene (93\%), while mutations in the ETFA (5\%) and ETFB (2\%) genes are the exceptions. Almost all patients with late-onset MADD (98\%) are clearly responsive to riboflavin.

Conclusions: Late-onset MADD is probably an underdiagnosed disease and should be considered in all patients with acute or chronic muscular symptoms or acute metabolic decompensation with hypoglycemia, acidosis, encephalopathy and hepatopathy. This may not only prevent patients from invasive diagnostic procedures such as muscle biopsies, but also help to avoid fatal metabolic decompensations.
\end{abstract}

Keywords: Glutaric aciduria type 2, Lipid storage myopathy, Muscle weakness, Metabolic decompensation, Riboflavin, ETFDH, ETFA, ETFB

\section{Background}

Multiple acyl-CoA dehydrogenase deficiency (MADD, MIM \#231680), also called glutaric aciduria type 2 (GA2), is an inborn error of metabolism affecting the oxidation of fatty acids as well as the catabolism of branched-chain amino acids, lysine and tryptophan. MADD is caused by deficiency of either an electron-transfer flavoprotein (ETF, encoded by ETFA and ETFB) or an electron-transfer flavoprotein dehydrogenase (ETFDH, encoded by ETFDH).

Correspondence: sarah.gruenert@uniklinik-freiburg.de

Center of Pediatrics and Adolescent Medicine, University Hospital Freiburg, Freiburg, Germany
Inheritance follows an autosomal recessive trait [1]. The metabolic defects result in impaired adenosine triphosphate (ATP) biosynthesis, excessive lipid accumulation in different organs and insufficient gluconeogenesis [1]. The clinical phenotype is heterogeneous and has been classified into three groups: neonatal onset with congenital anomalies (type 1), neonatal onset without anomalies (type 2), and mild and/or later onset (type 3) [1,2]. While neonates usually present with severe metabolic decompensations including metabolic acidosis, nonketotic hypoglycemia, hyperammonemia, hypotonia, coma and cardiomyopathy, the course and age at presentation 
of later-onset forms is extremely variable. In adolescents and adults, muscular or cardiac symptoms or episodic vomiting are usually first features suggestive for MADD [1,3-9]. Therapeutic management mostly comprises a diet restricted in fat and protein and the avoidance of fasting. The majority of patients respond well to riboflavin therapy.

Diagnosis is based on both the urinary organic acid profile and the acylcarnitine pattern in dried blood/ plasma. The characteristic urinary organic acid pattern comprises elevated levels of glutaric, ethylmalonic, 3-hydroxyisovaleric, 2-hydroxyglutaric, 5-hydroxyhexanoic, adipic, suberic, sebacic, and dodecanedioic acid without relevant ketonuria, especially if combined with glycine conjugates of $\mathrm{C} 4$ and $\mathrm{C} 5$ acids [1]. Acylcarnitine analysis usually reveals increased concentrations of several short-, medium- and long-chain acylcarnitines, such as C4, C5, C5-DC, C6, C8, C10, C12, C14:1, C16, C18:1 [10]. However, the diagnosis may be challenging in late-onset cases since the biochemical abnormalities may be mild, atypical or only detectable during metabolic decompensations. This report aims at providing a comprehensive description of late-onset MADD in its clinical and genetic heterogeneity by analysis of all cases published to date.

\section{Methods}

A systematic literature search in PubMed was performed using the terms "multiple acyl-CoA dehydrogenase deficiency", "glutaric aciduria type 2", "glutaric acidemia type 2", "ETFDH", "ETFA" and "ETFB" in order to obtain comprehensive information on the clinical course of MADD patients with late onset of the disease (type 3). Late onset was defined as onset beyond the neonatal period ( $>28$ days). The search was performed in January 2014. 350 cases of late-onset MADD published between 1979 and 2014 were identified (for detailed data and references see Additional file 1: Table S1). All cases were evaluated and analyzed with a special focus on the patients' age at onset, age at diagnosis, symptoms, biochemical abnormalities, affected genes and response to riboflavin treatment. Riboflavin responsiveness was defined as a positive clinical response to treatment with improvement of symptoms, not only biochemical parameters.

\section{Results}

350 cases of late-onset MADD were reviewed. Detailed information on all patients is displayed in the Additional file 1: Table S1 as well as in Table 1. 144 patients were female, 177 male, the sex of 29 individuals was not reported. Mean age at onset was 19.2 years $(n=273$, range 0.13-68 years) (Figure 1). Seven patients were identified by newborn screening, and another 5 patients were diagnosed by early investigations in the neonatal period due to an affected sibling. In 2 patients the diagnosis

$\begin{aligned} & \text { Table } \mathbf{1} \text { Clinical information on } \mathbf{3 5 0} \text { patients with } \\
& \text { late-onset MADD published in the literature }\end{aligned}$
\begin{tabular}{ll} 
Sex & $\begin{array}{l}\text { Female } n=144, \text { male } n=177 \\
\text { not reported } n=29\end{array}$ \\
Identified by newborn screening & $2.0 \%(7 / 350)$ patients \\
Identified by family screening & $1.4 \%(5 / 350)$ patients \\
Acute symptoms & $33.1 \%(111 / 335)$ of patients \\
Chronic symptoms & $85.3 \%(291 / 341)$ of patients \\
Acute and chronic symptoms & $20.4 \%(68 / 333)$ of patients \\
Mean age at onset of symptoms & 19.2 years $(n=273)$ \\
Mean diagnostic delay & 3.9 years (0-29 years) \\
Asymptomatic & $2.6 \%(9 / 349)$ of patients \\
Deceased patients & $5.2 \%(18 / 349)$ of patients \\
Riboflavin responsiveness & $98.4 \%(256 / 260)$ of patients \\
\hline
\end{tabular}

was only made post mortem. Mean age at diagnosis of symptomatic patients was 17.6 years $(n=111$, median 14.0 years, range $0.13-69$ years). The mean delay between onset of symptoms and diagnosis was 3.9 years $(n=93$, range 0-29 years). If only patients without acute decompensations were considered $(n=38)$, the mean latency was 3.7 years (range $0-29$ years). $33.1 \%$ of patients (111/335) displayed acute signs and symptoms, i.e., metabolic decompensations with hypoglycemia and acidosis, while chronic features were reported in $85.3 \%$ (291/341) of patients. Chronic manifestations mainly comprised muscular weakness, exercise intolerance and muscle pain. 20.4\% (68/333) had both, acute episodes and chronic symptoms. $5.2 \%(18 / 349)$ of patients had already died when reported in the literature (mean age at death 5.8 years, range $0.2-20$ years). Among those only the minority (4/14, 28.6\%) deceased during the initial metabolic crisis. In 5 of 11 patients with fatal outcome of whom detailed data were available, the diagnosis was known before the life-ending event. Three of those had been identified by newborn screening or early neonatal screening due to an affected older sibling while two patients had been diagnosed symptomatically in infancy/childhood. In the remaining 6 patients the diagnosis was only made during the fatal metabolic decompensation or post mortem. 2.6\% (9/349) of patients have remained asymptomatic until a mean age of 6.15 years $(0.5-14.0$ years).

Results of organic acid analysis in urine were available in 258 patients. A typical metabolite pattern was detected at least once in 236/258 patients (91.5\%) while only unremarkable organic acid results were reported in $22 / 258$ individuals (8.5\%). In some cases, elevated signals of only single metabolites such as ethylmalonic acid or glutaric acid were detected [11-13]. Some patients displayed a pathognomonic metabolite pattern only intermittently, i.e. during metabolic decompensations. In several patients, the initially detected biochemical abnormalities disappeared 


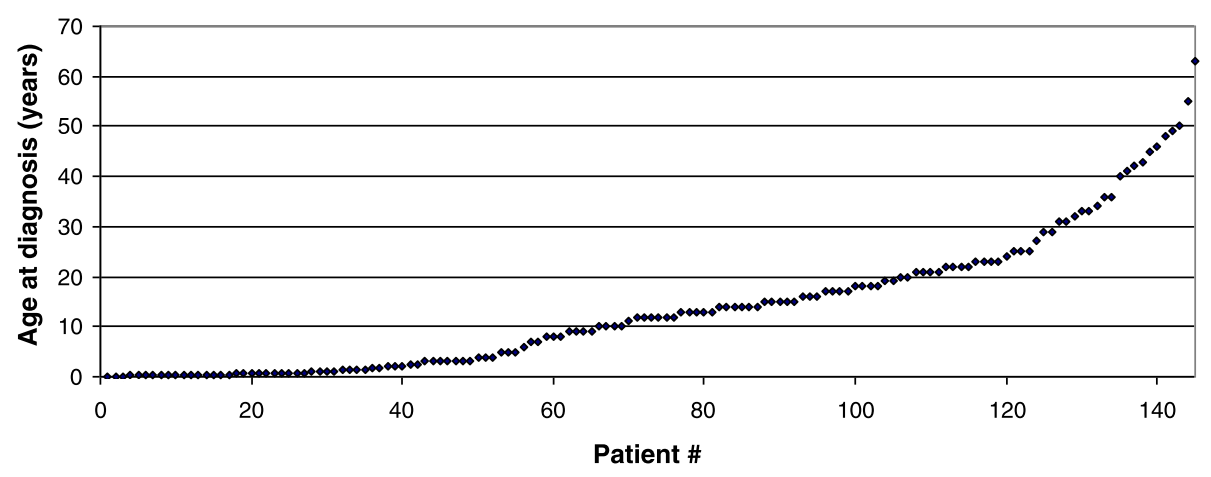

Figure 1 Age at onset in 146 late-onset MADD patients. Age at onset of symptoms ranges from early infancy to late adulthood. The mean age at onset of 146 patients with late-onset MADD of whom detailed information was given in the literature is 14.3 years. Of additional 56 cases reported by Wang et al. [55] and 71 cases reported by Xi et al. [28] only the mean age at onset (24.5 \pm 12.6 years with a range from 4 to 55 years and $25.0 \pm 13.3$ years with a range from 4 to 36 years, respectively) is known. If these cases are also taken into account, the mean age at onset in this study cohort is 19.2 years.

under treatment with riboflavin. Acylcarnitine profiles were at least intermittently characteristic for MADD in $201 / 214$ patients (93.9\%). In 13/214 individuals (6.1\%) only normal acylcarnitine profiles were reported. Again, in some patients an abnormal acylcarnitine pattern was only found during metabolic decompensations or after a prolonged exercise test.

Among 260 patients of whom data on riboflavin therapy were available, 256 (98.4\%) were reported to be clearly riboflavin-responsive, while in 2 patients efficacy of riboflavin therapy was only partial with merely "some improvement" of the muscular symptoms [14,15]. In one of these patients riboflavin supplementation was discontinued later because of limited efficacy [14]. In one other patient in whom riboflavin therapy was started during severe metabolic decompensation fatal outcome could not be prevented [16]. Only one patient was reported to be clearly unresponsive to riboflavin [17].

The genotype was reported of 245 patients. The vast majority carried mutations in the ETFDH gene $(228 / 245$, 93.1\%), while in only few patients ETFA and ETFB mutations were detected $(11 / 245,4.5 \%$ and $6 / 245,2.4 \%$ of patients, respectively) (Figure 2). In one patient, no mutation was identified in the ETFA and ETFB genes, while the $E T F D H$ gene was not studied. In 2 patients, no mutations could be delineated despite analyzing of the entire coding regions and flanking intronic sequences of the ETFA, ETFB and ETFDH genes [18,19].

\section{Discussion}

This evaluation of all published cases of late-onset MADD characterizes the clinical and genetic heterogeneity of this disorder with a special focus on typical symptoms, the diagnostic work-up and therapeutic options. The clinical phenotype of late-onset MADD is highly variable and ranges from acute, in some cases even fatal metabolic crises in infancy to asymptomatic adults. Even within the same family the clinical picture can vary profoundly [20,21]. Although most patients become symptomatic within the first two decades, onset of symptoms ranges from the second month of life to late adulthood. The vast majority of patients presented with chronic, mainly myopathic symptoms. Nevertheless, one third of patients

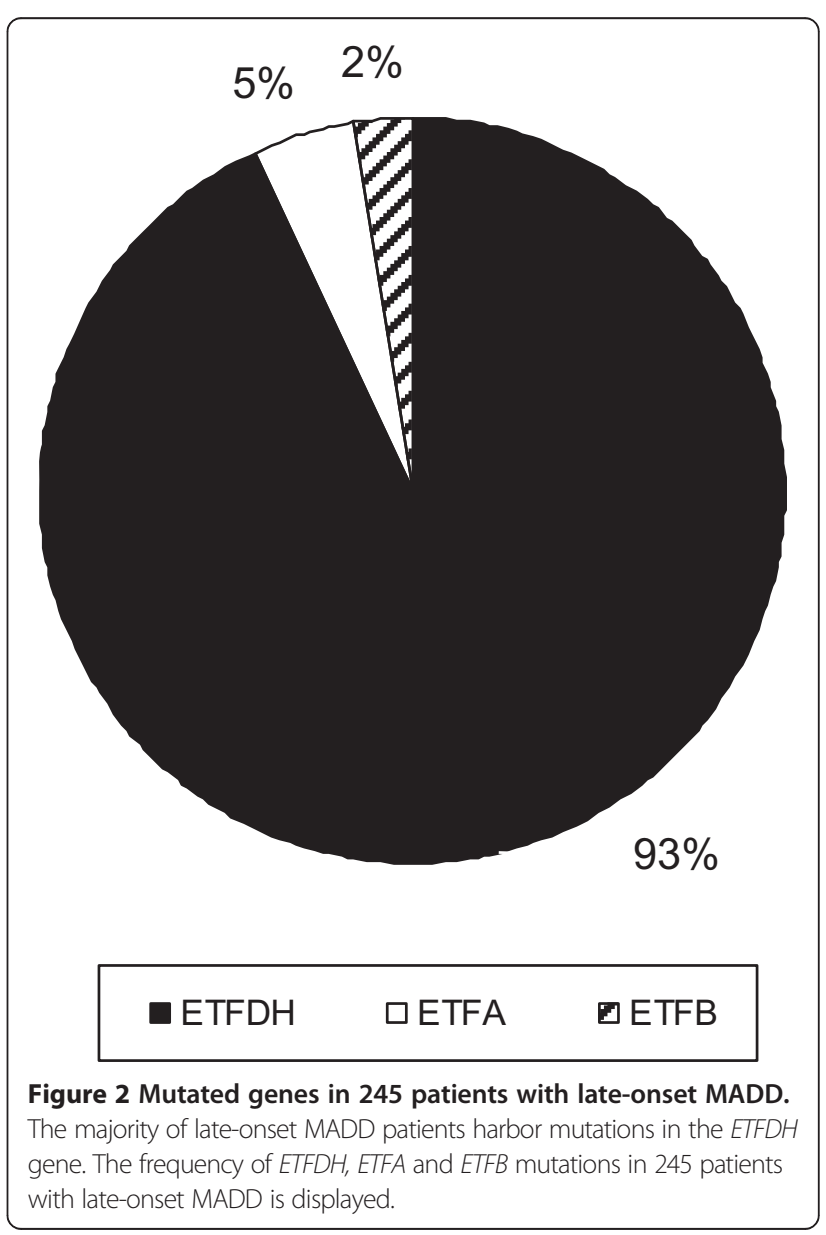


also displayed acute symptoms or metabolic decompensations which may be life-threatening if not treated adequately. Although these decompensations mostly occur in childhood, severe metabolic crises in adulthood have also been described [14,22-26]. Decompensations are characterized by acidosis, hypoglycemia, elevated activities of transaminases, rhabdomyolysis with raised creatine kinase activity, and, eventually, hyperammonemia. These episodes are usually triggered by catabolic states, either due to infections and febrile illnesses or to a reduced energy supply. However, in adulthood, other triggering factors like operations, low-energy diets or weight loss of other reasons, alcohol, valproate therapy, pregnancy or labor may also play a role $[9,15,24,27]$. Chronic features include exercise intolerance, muscle weakness, muscle wasting and muscular pain. Some cases may develop respiratory insufficiency $[13,23,28]$, while rhabdomyolysis is not often seen. Muscle biopsies usually reveal lipid storage myopathy (Figure 3). Especially in adult cases, diagnosis is often not straight-forward, resulting in a significant diagnostic delay. One patient has been diagnosed as late as 29 years after the onset of symptoms. Surprisingly, the diagnostic delay was not less pronounced in patients with acute symptoms. For patients with acute decompensations early diagnosis and treatment are essential for survival and a positive outcome. Because inborn errors of metabolism are commonly thought to be "pediatric diseases", awareness of these conditions among physicians treating adults is often low. Diagnostic work-up including MS/MS-based acylcarnitine profiling in dried blood spots/plasma and organic acid analysis in urine should be initiated promptly in every patient with acidosis of unknown origin. Additionally, MADD should be ruled out in any patient with suggestive muscular symptoms. As in patients with milder disease variants symptoms are often intermittent and only become evident during periods of illness and catabolic stress, late-onset MADD might be underdiagnosed [29,30]. Organic acid and acylcarnitine analyses should be performed as first-line investigations with a low degree of suspicion as they may prevent patients from more invasive investigations like liver or muscle biopsies.

Making a biochemical diagnosis of late-onset MADD may be challenging in both symptomatic and asymptomatic individuals [31]. Most patients display a typical pattern of organic acids in urine and acylcarnitines in dried blood/plasma at least during metabolic decompensations. However, organic acid and acylcarnitine results may be unremarkable especially during metabolically stable conditions and in some cases even during catabolism $[7,9,13,19,31-36]$. Others show only selected abnormal metabolites, but not a full diagnostic pattern. Some therapeutic interventions like MCT oil, valproic acid or particular antibiotics (pivalic acid) can also mimic at least parts of the biochemical profile [37,38]. Finally, due to common polymorphic alleles in the ACADS gene about $14 \%$ of the population will excrete ethylmalonic acid or will have elevated C4-acylcarnitine species in plasma [39]. This indicates, that MADD cannot be definitely excluded by a single metabolic screening test. The diagnosis should therefore rely on blood and urine analysis and ultimately be confirmed by molecular methods. In patients with muscular symptoms and histologically proven lipid storage myopathy but unremarkable metabolite patterns molecular analysis (starting with the ETFDH gene, followed by sequencing of ETFA and ETFB) is advisable.

Recently, several riboflavin transporters have been identified [40-42]: SLC52A1 (RFT1), SLC52A2 (RFT3) and SLC52A3 (RFT2) are transmembrane proteins that mediate the cellular uptake of riboflavin [42]. Defects of these transporters lead to cellular riboflavin deficiency and may result in biochemical and clinical abnormalities mimicking MADD $[43,44]$. Only one patient with a RFT1 defect has been identified so far [43]. This woman was clinically asymptomatic, but showed biochemical evidence of riboflavin deficiency. She was diagnosed after her daughter had presented with poor suck, hypoglycemia, and metabolic acidosis within the first days of life [45]. Other riboflavin transporter defects that can mimick mild MADD biochemically are the Brown-Vialetto-Van Laere (BVVL) and the Fazio Londe (FL) syndrome, two disorders which are nowadays considered to be the same disease entity $[44,46,47]$. These cases are due to mutations in the SLC52A2 or SLC52A3 genes which code for the RFT3 and RFT2 proteins, respectively. The clinical picture of these rare neurologic disorders usually differs from MADD and comprises sensorineural deafness (not in Fazio Londe syndrome), bulbar palsy, facial weakness and respiratory distress [44,47]. However, a certain clinical overlap, especially with respect to the muscular symptoms, can make it difficult to distinguish late-onset MADD and BVVL/FL syndrome. It is well possible that some patients with genetic riboflavin transporter defects have been misdiagnosed with MADD. In particular, patients in whom no mutations in ETFDH, ETFA and $E T F B$ can be identified should be investigated for a defective riboflavin transporter.

The positive clinical effects of riboflavin treatment are well documented as are the molecular mechanisms for riboflavin responsiveness $[4,7,8,29,48,49]$. In particular, the chaperone effects that can compensate for inherited folding defects of ETFDH, have recently been described [49]. The vast majority of late-onset MADD patients respond very well to riboflavin. It has been shown that ETFDH deficiency may be associated with secondary coenzyme Q10 (Q10) deficiency. Thus, Q10 supplementation in addition to riboflavin treatment has been suggested [50]. The finding of ETFDH mutations in patients with the 

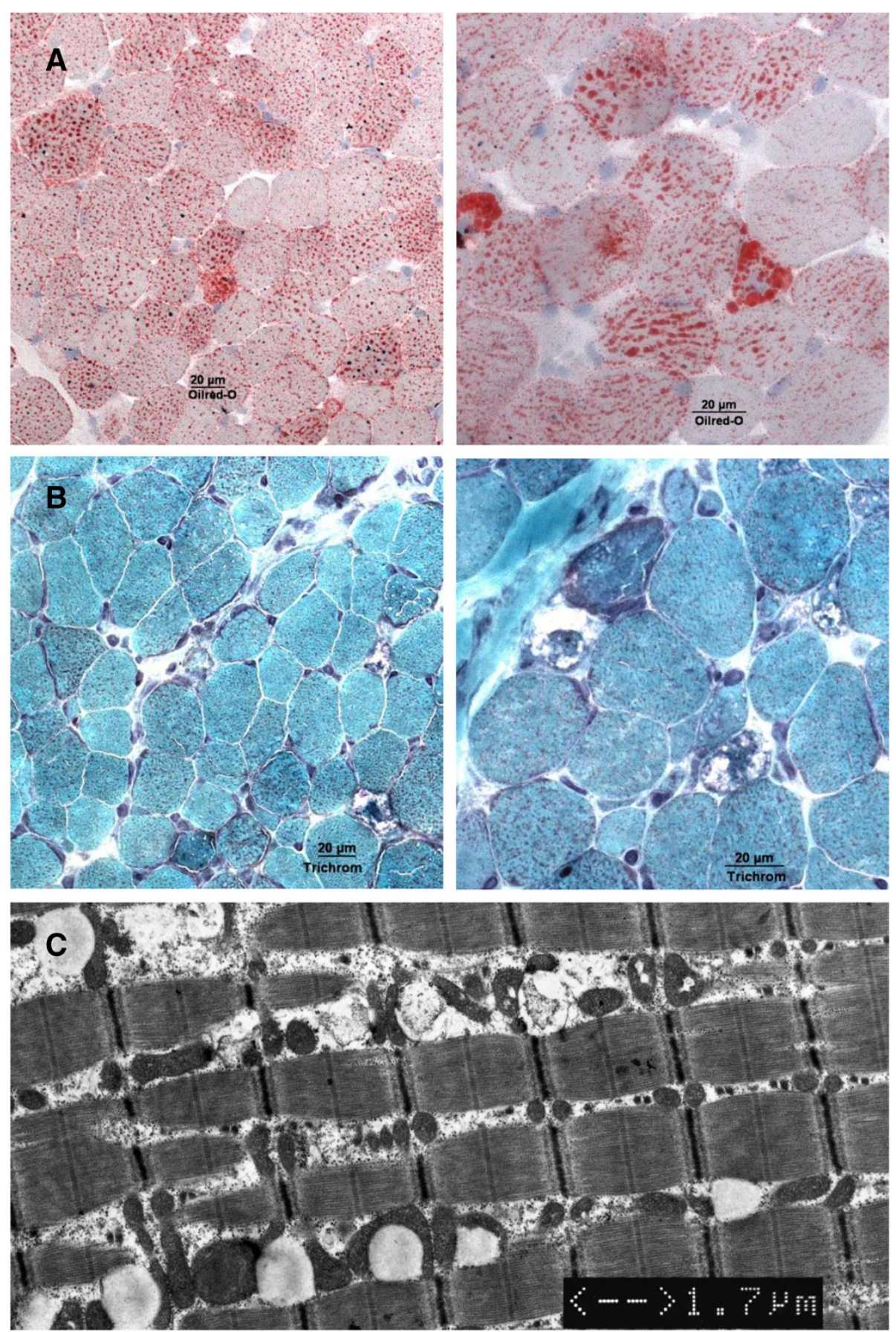

Figure 3 Lipid storage myopathy. Histological picture of lipid storage myopathy typical for late-onset MADD. A Oilred stain. B Trichrome stain C Electron microscopy.

myopathic phenotype of Q10 deficiency initially lead to the suggestion that the late-onset form of MADD and the myopathic form of Q10 deficiency are allelic diseases [50]. However, in the meanwhile, late-onset MADD patients without Q10 deficiency have been reported contradicting this hypothesis [16]. Q10 deficiency has been found to be associated with increased mitochondrial production of reactive oxygen species (ROS) due to an electron leak of misfolded variant ETFDH proteins with impaired Q10 binding affinity $[49,51]$. Based on studies with fibroblasts of six patients with riboflavin-responsive MADD Cornelius et al. have reported that Q10 treatment can decrease ROS production and may relieve oxidative stress. This suggests that late-onset MADD patients could benefit from a 
combined treatment of riboflavin and Q10 [52]. Q10 treatment alone is not advisable. Six Chinese patients, who were given Q10 alone before the diagnosis of late-onset MADD was made, showed only a mild response with their muscle strength never returning to normal [28]. Yamaguchi et al. studied the therapeutic effect of bezafibrate by an in vitro probe acylcarnitine assay using cultured fibroblasts of a patient with late-onset MADD [53]. The aberrantly elevated MADD-characteristic acylcarnitines were clearly corrected by the presence of bezafibrate in culture medium. Following these results, a clinical trial of bezafibrate was performed in the same patient, a 2-year-old boy, and resulted in a dramatical improve of his motor and cognitive skills in combination with a reduction of $\mathrm{C} 4, \mathrm{C} 8, \mathrm{C} 10$ and $\mathrm{C} 12$ acylcarnitines in blood and a normalization of the urinary organic acid profile. Notably, this boy has never been treated with riboflavin. Due to the loss of carnitine conjugates via the urine, MADD patients are prone to carnitine deficiency, which may require oral carnitine supplementation [5,9]. Taken together, the mainstay of therapy in patients with late-onset MADD is riboflavin with excellent outcome data, and supplementation should be prompted immediately after diagnosis.

A clear genotype-phenotype correlation has been described for the different subtypes of MADD [54]: Homozygosity for null mutations is usually associated with MADD type 1 , whereas even minor amounts of residual ETF/ETFDH activity seem to be sufficient to prevent embryonic development of congenital anomalies giving rise to type 2 disease. A relatively high residual activity is found in the late-onset form type 3 [54]. All patients with late-onset MADD of whom mutation data are published carry at least one missense variation, which may potentially result in some residual activity (Additional file 1: Table S1). The genotype-phenotype correlation within the group of late-onset patients, however, is poor. Apart from the disease-causing mutation other, especially exogenous, factors such as febrile infections, a restricted diet and other physiologic stressors may play an important role in the modulation of the clinical phenotype. Most mutations seem to be private. Three common mutations in the ETFDH gene have been described which are mainly found in the Chinese and Taiwanese population: c.250G > A (p.A84T), c.770A > G (p.Y257C), c.1227A > C (p.L409F) $[23,28,55]$. As this literature review has shown, the vast majority of late-onset patients harbour mutations in the ETFDH gene, while ETFA or ETFB mutations are found in only about $7 \%$ of individuals. Therefore, mutation analysis in this cohort should first focus on ETFDH.

While early-onset MADD is a disease with high mortality, the prognosis of late-onset MADD seems to be good. Nevertheless, $5 \%$ of patients reported in the literature had died mainly during metabolic decompensations. In several patients, death could not be prevented despite the known diagnosis of MADD. Although most deaths occurred in childhood, several cases with fatal outcome during adolescence and adulthood have been described $[22,56]$. Even patients who present with chronic symptoms in adulthood are prone to metabolic crises. To prevent unfavorable outcomes, patients need to be aware of the disease risks and potential triggers of decompensations. MADD represents an official target of newborn screening in only few countries, e.g. in the United States. Limited data exist on the detection rate of late-onset MADD by this approach. However, based on the number of detected cases so far, it is quite possible that most MADD patients are recognized by newborn screening [57]. Early diagnosis and treatment from the neonatal period might modify the clinical outcome and possibly even prevent symptoms in late-onset MADD patients.

In conclusion, late-onset MADD may be an underdiagnosed disease in adults with myopathy and should be considered early in the differential diagnosis. This may not only prevent patients from invasive diagnostic procedures such as muscle biopsies, but also help to avoid fatal metabolic decompensations.

\section{Additional file}

Additional file 1: Table S1. Clinical, biochemical and molecular data of 350 patients with late-onset multiple acyl-CoA dehydrogenase deficiency published in the literature between 1979 and 2014. Patient numbers in this supplemental table do not correspond to those displayed in Figure 1.

\section{Competing interests}

The author has no competing interests to declare.

\section{Acknowledgement}

I am grateful to Prof. Dr. U. Spiekerkötter, Center of Pediatrics and Adolescent Medicine, University Hospital Freiburg, Germany, and Prof. Dr. J.O. Sass, Division of Clinical Chemistry and Biochemistry, University Children's Hospital Zürich, Switzerland, for the valuable discussions and their critical revision of the manuscript. I also thank PD Dr. Janbernd Kirschner, Center of Pediatrics and Adolescent Medicine, University Hospital Freiburg, Germany, for the muscle histology pictures displayed in Figure 3.

Parts of this study have been presented as a poster at the Annual Meeting of the Working Group for Pediatric Metabolic Disorders, March 2013, Fulda, Germany and have been published as an abstract in the Monatsschrift Kinderheilkunde (Monatsschr Kinderheilkd 2013;161:279). For this presentation, the "Ursula-Wachtel-Preis" of the German Working Group for Pediatric Metabolic Disorders was awarded to Sarah C. Grünert.

Received: 28 April 2014 Accepted: 8 July 2014

Published: 22 July 2014

\section{References}

1. Frerman FE, Goodman SI: Defects of Electron Transfer Flavoprotein and Electron Transfer Flavoprotein-Ubiquinone Oxidoreductase: Glutaric Acidemia Type II. In The Metabolic and Molecular Bases of Inherited Disease. Edited by Scriver CR, Sly WS, Childs B, Beaudet AL, Valle D, Kinzler KW, Vogelstein B; 2001

2. Goodman SI, Frerman FE: Glutaric acidaemia type II (multiple acyl-CoA dehydrogenation deficiency). J Inherit Metab Dis 1984, 7(Suppl 1):33-37. 
3. Dusheiko G, Kew MC, Joffe BI, Lewin JR, Mantagos S, Tanaka K: Recurrent hypoglycemia associated with glutaric aciduria type II in an adult. $N$ Engl J Med 1979, 301(26):1405-1409.

4. Bell RB, Brownell AK, Roe CR, Engel AG, Goodman SI, Frerman FE, Seccombe DW, Snyder FF: Electron transfer flavoprotein: ubiquinone oxidoreductase (ETF:QO) deficiency in an adult. Neurology 1990, 40(11):1779-1782.

5. Izumi R, Suzuki N, Nagata M, Hasegawa T, Abe Y, Saito Y, Mochizuki H, Tateyama M, Aoki M: A case of late onset riboflavin-responsive multiple acyl-CoA dehydrogenase deficiency manifesting as recurrent rhabdomyolysis and acute renal failure. Intern Med 2011, 50(21):2663-2668.

6. Sugai F, Baba K, Toyooka K, Liang WC, Nishino I, Yamadera M, Sumi H, Fujimura $\mathrm{H}$, Nishikawa Y: Adult-onset multiple acyl CoA dehydrogenation deficiency associated with an abnormal isoenzyme pattern of serum lactate dehydrogenase. Neuromuscul Disord 2012, 22(2):159-161.

7. Wasant P, Kuptanon C, Vattanavicharn N, Liammongkolkul S, Ratanarak P Sangruchi T, Yamaguchi S: Glutaric aciduria type 2, late onset type in Thai siblings with myopathy. Pediatr Neurol 2010, 43(4):279-282.

8. Ishii K, Komaki H, Ohkuma A, Nishino I, Nonaka I, Sasaki M: Central nervous system and muscle involvement in an adolescent patient with riboflavin-responsive multiple acyl-CoA dehydrogenase deficiency. Brain Dev 2010, 32(8):669-672.

9. Mareska MC, Adams KK, Muenzer J, Frerman F, Braun TG, Howard JF Jr: Adult-onset presentation of Glutaric Acidemia Type II with Myopathy. J Clin Neuromuscul Dis 2003, 4(3):124-128.

10. Morris AAM, Spiekerkoetter U: Disorders of Mitochondrial Fatty Acid Oxidation and Related Metabolic Pathways. In Inborn Metabolic Diseases, Diagnosis and Treatment. Edited by Saudubray J-M, van den Berghe G, Walter JH. New York: Springer-Verlag Berlin Heidelberg New York; 2012:201-212.

11. Amendt BA, Rhead WJ: The multiple acyl-coenzyme A dehydrogenation disorders, glutaric aciduria type II and ethylmalonic-adipic aciduria. Mitochondrial fatty acid oxidation, acyl-coenzyme A dehydrogenase, and electron transfer flavoprotein activities in fibroblasts. J Clin Invest 1986, 78(1):205-213

12. Harpey JP, Charpentier C, Coude M, Divry P, Paturneau-Jouas M: Sudden infant death syndrome and multiple acyl-coenzyme A dehydrogenase deficiency, ethylmalonic-adipic aciduria, or systemic carnitine deficiency. J Pediatr 1987, 110(6):881-884.

13. Russell AP, Schrauwen P, Somm E, Gastaldi G, Hesselink MK, Schaart G, Kornips E, Lo SK, Bufano D, Giacobino JP, Muzzin P, Ceccon M, Angelini C, Vergani L: Decreased fatty acid beta-oxidation in riboflavin-responsive, multiple acylcoenzyme A dehydrogenase-deficient patients is associated with an increase in uncoupling protein-3. J Clin Endocrinol Metab 2003, 88(12):5921-5926.

14. Grice AS, Peck TE: Multiple acyl-CoA dehydrogenase deficiency: a rare cause of acidosis with an increased anion gap. Br J Anaesth 2001, 86(3):437-441.

15. Vergani $L$, Barile $M$, Angelini $C$, Burlina AB, Nijtmans $L$, Freda MP, Brizio $C$ Zerbetto E, Dabbeni-Sala F: Riboflavin therapy. Biochemical heterogeneity in two adult lipid storage myopathies. Brain 1999, 122(Pt 12):2401-2411.

16. Liang WC, Ohkuma A, Hayashi YK, Lopez LC, Hirano M, Nonaka I, Noguchi S, Chen LH, Jong YJ, Nishino I: ETFDH mutations, CoQ10 levels, and respiratory chain activities in patients with riboflavin-responsive multiple acyl-CoA dehydrogenase deficiency. Neuromuscul Disord 2009, 19(3):212-216.

17. Burns SP, Holmes HC, Chalmers RA, Johnson A, lles RA: Proton NMR spectroscopic analysis of multiple acyl-CoA dehydrogenase deficiency-capacity of the choline oxidation pathway for methylation in vivo. Biochim Biophys Acta 1998, 1406(3):274-282.

18. Cotelli MS, Vielmi V, Rimoldi M, Rizzetto M, Castellotti B, Bertasi V, Todeschini A, Gregorelli V, Baronchelli C, Gellera C, Padovani A, Filosto M: Riboflavin-responsive multiple acyl-CoA dehydrogenase deficiency with unknown genetic defect. Neurol Sci 2012, 33(6):1383-1387.

19. Wen B, Dai T, Li W, Zhao Y, Liu S, Zhang C, Li H, Wu J, Li D, Yan C: Riboflavin-responsive lipid-storage myopathy caused by ETFDH gene mutations. J Neurol Neurosurg Psychiatry 2010, 81(2):231-236.

20. Law LK, Tang NL, Hui J, Fung SL, Ruiter J, Wanders RJ, Fok TF, Lam CW: Novel mutations in ETFDH gene in Chinese patients with riboflavin-responsive multiple acyl-CoA dehydrogenase deficiency. Clin Chim Acta 2009, 404(2):95-99.

21. Schiff M, Froissart R, Olsen RK, Acquaviva C, Vianey-Saban C: Electron transfer flavoprotein deficiency: functional and molecular aspects. Mol Genet Metab 2006, 88(2):153-158.
22. Fitzgerald M, Crushell E, Hickey C: Cyclic vomiting syndrome masking a fatal metabolic disease. Eur J Pediatr 2013, 172(5):707-710.

23. Lan MY, Fu MH, Liu YF, Huang CC, Chang YY, Liu JS, Peng CH, Chen SS: High frequency of ETFDH c.250G > A mutation in Taiwanese patients with late-onset lipid storage myopathy. Clin Genet 2010, 78(6):565-569.

24. Triggs WJ, Roe CR, Rhead WJ, Hanson SK, Lin SN, Willmore L: Neuropsychiatric manifestations of defect in mitochondrial beta oxidation response to riboflavin. J Neurol Neurosurg Psychiatry 1992, 55(3):209-211.

25. Scheicht D, Werthmann ML, Zeglam S, Holtmeier J, Holtmeier W, Strunk J: [Muscle weakness and early stages of liver failure in a 22-year-old man]. Internist (Berl) 2013, 54(8):1016-1022.

26. Olsen RK, Olpin SE, Andresen BS, Miedzybrodzka ZH, Pourfarzam M, Merinero B, Frerman FE, Beresford MW, Dean JC, Cornelius N, Andersen O, Oldfors A, Holme E, Gregersen N, Turnbull DM, Morris AA: ETFDH mutations as a major cause of riboflavin-responsive multiple acyl-CoA dehydrogenation deficiency. Brain 2007, 130(Pt 8):2045-2054.

27. Papadimitriou A, Servidei S: Late onset lipid storage myopathy due to multiple acyl CoA dehydrogenase deficiency triggered by valproate. Neuromuscul Disord 1991, 1(4):247-252.

28. Xi J, Wen B, Lin J, Zhu W, Luo S, Zhao C, Li D, Lin P, Lu J, Yan C: Clinical features and ETFDH mutation spectrum in a cohort of 90 Chinese patients with late-onset multiple acyl-CoA dehydrogenase deficiency. J Inherit Metab Dis 2013, 37:399-404

29. Henriques BJ, Rodrigues JV, Olsen RK, Bross P, Gomes CM: Role of flavinylation in a mild variant of multiple acyl-CoA dehydrogenation deficiency: a molecular rationale for the effects of riboflavin supplementation. J Biol Chem 2009, 284(7):4222-4229.

30. Koppel S, Gottschalk J, Hoffmann GF, Waterham HR, Blobel H, Kolker S: Late-onset multiple acyl-CoA dehydrogenase deficiency: a frequently missed diagnosis? Neurology 2006, 67(8):1519.

31. Pollard LM, Williams NR, Espinoza L, Wood TC, Spector EB, Schroer RJ, Holden KR: Diagnosis, treatment, and long-term outcomes of late-onset (type III) multiple acyl-CoA dehydrogenase deficiency. J Child Neurol 2010, 25(8):954-960.

32. Kaminsky P, Acquaviva-Bourdain C, Jonas J, Pruna L, Chaloub GE, Rigal O, Grignon Y, Vianey-Saban C: Subacute myopathy in a mature patient due to multiple acyl-coenzyme A dehydrogenase deficiency. Muscle Nerve 2011, 43(3):444-446.

33. Mumtaz HA, Gupta V, Singh P, Marwaha RK, Khandelwal N: MR imaging findings of glutaric aciduria type II. Singapore Med J 2010, 51(4):e69-71.

34. Takken T, Custers J, Visser G, Dorland L, Helders P, de Koning T: Prolonged exercise testing in two children with a mild Multiple Acyl-CoA-Dehydrogenase deficiency. Nutr Metab (Lond) 2005, 2(1):12.

35. Gianazza E, Vergani L, Wait R, Brizio C, Brambilla D, Begum S, Giancaspero TA, Conserva F, Eberini I, Bufano D, Angelini C, Pegoraro E, Tramontano A, Barile M: Coordinated and reversible reduction of enzymes involved in terminal oxidative metabolism in skeletal muscle mitochondria from a riboflavin-responsive, multiple acyl-CoA dehydrogenase deficiency patient. Electrophoresis 2006, 27(5-6):1182-1198.

36. Van Hove JL, Grunewald S, Jaeken J, Demaerel P, Declercq PE, Bourdoux P, Niezen-Koning K, Deanfeld JE, Leonard JV: D, L-3-hydroxybutyrate treatment of multiple acyl-CoA dehydrogenase deficiency (MADD). Lancet 2003, 361(9367):1433-1435.

37. Boemer F, Schoos R, de Halleux V, Kalenga M, Debray FG: Surprising causes of C5-carnitine false positive results in newborn screening. Mol Genet Metab 2014, 111(1):52-54.

38. Abdenur JE, Chamoles NA, Guinle AE, Schenone AB, Fuertes AN: Diagnosis of isovaleric acidaemia by tandem mass spectrometry: false positive result due to pivaloylcarnitine in a newborn screening programme. J Inherit Metab Dis 1998, 21(6):624-630.

39. Corydon MJ, Vockley J, Rinaldo P, Rhead WJ, Kjeldsen M, Winter V, Riggs C, Babovic-Vuksanovic D, Smeitink J, De Jong J, Levy H, Sewell AC, Roe C, Matern D, Dasouki M, Gregersen N: Role of common gene variations in the molecular pathogenesis of short-chain acyl-CoA dehydrogenase deficiency. Pediatr Res 2001, 49(1):18-23.

40. Yonezawa A, Masuda S, Katsura T, Inui K: Identification and functional characterization of a novel human and rat riboflavin transporter, RFT1. Am J Physiol Cell Physiol 2008, 295(3):C632-641.

41. Yamamoto S, Inoue K, Ohta KY, Fukatsu R, Maeda JY, Yoshida Y, Yuasa H: Identification and functional characterization of rat riboflavin transporter 2. J Biochem 2009, 145(4):437-443. 
42. Yao $Y$, Yonezawa A, Yoshimatsu $H$, Masuda S, Katsura T, Inui K: Identification and comparative functional characterization of a new human riboflavin transporter hRFT3 expressed in the brain. J Nutr 2010, 140(7):1220-1226.

43. Ho G, Yonezawa A, Masuda S, Inui K, Sim KG, Carpenter K, Olsen RK, Mitchell JJ, Rhead WJ, Peters G, Christodoulou J: Maternal riboflavin deficiency, resulting in transient neonatal-onset glutaric aciduria Type 2 , is caused by a microdeletion in the riboflavin transporter gene GPR172B. Hum Mutat 2011 32(1):E1976-1984.

44. Bosch AM, Abeling NG, ljlst L, Knoester $H$, van der Pol WL, Stroomer AE, Wanders RJ, Visser G, Wijburg FA, Duran M, Waterham HR: Brown-Vialetto-Van Laere and Fazio Londe syndrome is associated with a riboflavin transporter defect mimicking mild MADD: a new inborn error of metabolism with potential treatment. J Inherit Metab Dis 2011, 34(1):159-164.

45. Chiong MA, Sim KG, Carpenter K, Rhead W, Ho G, Olsen RK, Christodoulou J: Transient multiple acyl-CoA dehydrogenation deficiency in a newborn female caused by maternal riboflavin deficiency. Mol Genet Metab 2007, 92(1-2):109-114

46. Dipti $\mathrm{S}$, Childs AM, Livingston JH, Aggarwal AK, Miller M, Williams C, Crow YJ: Brown-Vialetto-Van Laere syndrome; variability in age at onset and disease progression highlighting the phenotypic overlap with Fazio-Londe disease. Brain Dev 2005, 27(6):443-446.

47. Bosch AM, Stroek K, Abeling NG, Waterham HR, ljist L, Wanders RJ: The Brown-Vialetto-Van Laere and Fazio Londe syndrome revisited: natural history, genetics, treatment and future perspectives. Orphanet J Rare Dis 2012, 7:83.

48. Rosa M, Pascarella A, Parenti G, Buono S, Romano A, Della Casa R, Andria G, Marino M, Riccio MP, Bravaccio C: Developmental evolution in a patient with multiple acyl-coenzymeA dehydrogenase deficiency under pharmacological treatment. Eur J Paediatr Neurol 2012, 16(2):203-205.

49. Cornelius N, Frerman FE, Corydon TJ, Palmfeldt J, Bross P, Gregersen N Olsen RK: Molecular mechanisms of riboflavin responsiveness in patients with ETF-QO variations and multiple acyl-CoA dehydrogenation deficiency. Hum Mol Genet 2012, 21(15):3435-3448.

50. Gempel K, Topaloglu H, Talim B, Schneiderat P, Schoser BG, Hans VH, Palmafy B, Kale G, Tokatli A, Quinzii C, Hirano M, Naini A, DiMauro S, Prokisch H, Lochmüller H, Horvath R: The myopathic form of coenzyme Q10 deficiency is caused by mutations in the electron-transferring-flavoprotein dehydrogenase (ETFDH) gene. Brain 2007, 130(Pt 8):2037-2044.

51. Cornelius N, Byron C, Hargreaves I, Guerra PF, Furdek AK, Land J, Radford WW Frerman F, Corydon TJ, Gregersen N, Olsen RK: Secondary coenzyme Q10 deficiency and oxidative stress in cultured fibroblasts from patients with riboflavin responsive multiple Acyl-CoA dehydrogenation deficiency. Hum Mol Genet 2013, 22(19):3819-3827.

52. Cornelius N, Corydon TJ, Gregersen N, Olsen RK: Cellular consequences of oxidative stress in riboflavin responsive multiple acyl-CoA dehydrogenation deficiency patient fibroblasts. Hum Mol Genet 2014, Epub ahead of print.

53. Yamaguchi S, Li H, Purevsuren J, Yamada K, Furui M, Takahashi T, Mushimoto Y, Kobayashi H, Hasegawa Y, Taketani T, Fukao T, Fukuda S: Bezafibrate can be a new treatment option for mitochondrial fatty acid oxidation disorders: evaluation by in vitro probe acylcarnitine assay. Mol Genet Metab 2012, 107(1-2):87-91.

54. Olsen RK, Andresen BS, Christensen E, Bross P, Skovby F, Gregersen N: Clear relationship between ETF/ETFDH genotype and phenotype in patients with multiple acyl-CoA dehydrogenation deficiency. Hum Mutat 2003, 22(1):12-23.

55. Wang ZQ, Chen XJ, Murong SX, Wang N, Wu ZY: Molecular analysis of 51 unrelated pedigrees with late-onset multiple acyl-CoA dehydrogenation deficiency (MADD) in southern China confirmed the most common ETFDH mutation and high carrier frequency of c.250G >A. J Mol Med (Berl) 2011, 89(6):569-576.

56. Lee HC, Lai CK, Siu TS, Yuen YP, Chan KY, Chan AY, Tam S, Mak CM, Lam CW: Role of postmortem genetic testing demonstrated in a case of glutaric aciduria type II. Diagn Mol Pathol 2010, 19(3):184-186.
57. McHugh D, Cameron CA, Abdenur JE, Abdulrahman M, Adair O, Al Nuaimi SA, Ahlman H, Allen JJ, Antonozzi I, Archer S, Au S, Auray-Blais C, Baker M, Bamforth F, Beckmann K, Pino GB, Berberich SL, Binard R, Boemer F, Bonham J, Breen NN, Bryant SC, Caggana M, Caldwell SG, Camilot M, Campbell C, Carducci C, Bryant SC, Caggana M, Caldwell SG, et al: Clinical validation of cutoff target ranges in newborn screening of metabolic disorders by tandem mass spectrometry: a worldwide collaborative project. Genet Med 2011, 13(3):230-254.

doi:10.1186/s13023-014-0117-5

Cite this article as: Grünert: Clinical and genetical heterogeneity of late-onset multiple acyl-coenzyme A dehydrogenase deficiency. Orphanet Journal of Rare Diseases 2014 9:117.

\section{Submit your next manuscript to BioMed Central and take full advantage of:}

- Convenient online submission

- Thorough peer review

- No space constraints or color figure charges

- Immediate publication on acceptance

- Inclusion in PubMed, CAS, Scopus and Google Scholar

- Research which is freely available for redistribution

Submit your manuscript at www.biomedcentral.com/submit
C BioMed Central 